Ann. Zootech., I976, 25 (I), I43-I48.

NOTE

\title{
CONSÉQUENCES D'UNE DÉFICIENCE DU RÉGIME EN TRYPTOPHANE GHEZ LE PORC SUR LE NIVEAU D'INGESTION ET LES PERFORMANCES DE CROISSANCE
}

\author{
Y. HENRY et Barbara PASTISZRWSLA* \\ Station de Recherche's sur l'Elevage des Pores, \\ Centre national de Recherches sootechniques, I. N. R.A. \\ i8350 Jouy in Josas \\ * Polish Acadomy of Sciences. \\ Institute of Animal Physiology and Nutrition, \\ 0.5110 Jablonna near IIarsaz' (Polognc)
}

\section{RÉSTMÉ}

Iu cours d'une expérience d'une durée de six semaines, réalisée sur des porcs en croissance d'un poids vif initial de $20,6 \mathrm{~kg}$, nourric à volonté, l'administration d'un régime semi-puritié a base de tourteau d'arachide et de gluten de maiss, convenablement supplémenté en lysine, a permis de mettre en évidence une déficience en tryptophane, entrainant une dépression du niveau d'ingestion at des performances de croissance, accompagnée d'une molification du profil de l'aninoacidénie libre sanguine.

\section{INTRODLCTION}

I la suite de nombreux travaux réalisés sur le Rat (HARper, rg64) et sur le Poulet (ZIMMERMx et ScoTt, 1965$)$, il est maintenant établi que les diftérents cas de déséquilibre en acides aminés dans la ration retentissent d'unc manière défavorable sur le niveau spontané d'ingestion de nourriture, et cle là sur la vitesse de croissance et l'efficacité alimentaire. En ce qui concerne le Porc, les informations sur ce sujet sont relativement limitées. Certaines observations ont permis cependant de faire apparaitre les eflets néfastes d'un apport excessif de protéines (SUGAHARA 
't al.: I969) comme d'un acide aminé particulier, lorsque celui-ci n'est pa; (ou n'est plus) le facteur limitant du régime (Müllek et al, , r 967 ).

Des études récentes ché le Porc en croissance (MAHAx tt al., I973) ont montré par ailleurs que le tryptophane constitue généralement le facteur limitant secondaire, après la lysine, des rations à base de protéines végétales, du type maïs-tourteau de soja. La présent travail, réalisé sur porcs en croissance nourris à volonté, avait précisément pour objet de mettrc en évidence l'effet dépressif d'une carence en tryptophane sur le niveau de consommation et les performances de croissance, dans le cas d'un régime normalement supplémenté en lýsine.

\section{MATÉRIEL ET MÉTHODES}

Vingt porcs femelles de race Large White, d'un poids noyen initial de $20,6 \mathrm{~kg}$ ct élevés en loges individuelles, sont répartis en + lots de 5 animaux suivant la méthode des blocs, compte tenu du poids et de l'âge.

Les animaux du lot I reçoivent un régime de base (RB) semi-purifié ( $\left.{ }^{1}\right)$ renfermant 16 p. 100 le matières azotées. Ces dernières sont fournies pour moitié par du tourteau d'arachicle et çu gluten de maïs, de manière à induire un important déficit en lysine dont le taux est fixé à 0,40 p. 100 de la ration, pour une valeur en énergie digestible le l'ordre de 3 too kcalories par $\mathrm{kg}$ d'aliment frais. En ce qui concerne les autres acides aminés indispensables, les teneurs en théonine st en tryptophane peuvent être considérées comme marginales relativement aux recommandations habituelles (N. R. C., I 973), soit respectivement 0,50 et o, I 3 p. roo de la ration. La tencur en tryptophane a fait l'objet d'une estimation à partir des valeurs proposées pour des matières premières présentant des caractéristiques voisines (PION, communication personnelle). Dans le lot 2, le régime de base est supplémenté en l-lysine à la dose de 0,35 p. roo, tandis que dans le: lots 3 et 4 on procède aux supplémentations suivantes :

$$
\begin{aligned}
& \text { - lot } 3: 0,35 \text { p. I } 00 \text { 1-lysine ; } 0,05 \text { p. I oo l-thréonine: } \\
& \text {-. lot }+: 0.35 \text { p. } 100 \text { l-lysine }+0,03 \text { p. } 100 \text { l-tryptophane. }
\end{aligned}
$$

Dans chacun des lots, les régimes sont distribués à volonté sous forme de granulés de $5 \mathrm{~mm}$ de cliamètre, l'eau cle boisson étant laissée à discrétion à l'aide d'un abreuvoir automatique. Les consommations de nourriture sont enregistrées quotidiennement et les animax sont pesés une fois par semaine.

A l'issue des 6 semaines d'expérience, des prélèvements (le sang ( $10 \mathrm{ml}$ environ par animal) sont effectués à la veine cave antérieure 8 heures après le repas du matin, en v'ue de lá mesure do l'aminoacidémie libre. Pour cela, un échantillon moyen pondéré par lot est constitué à partir des 5 prélèvements individucls.

Les teneurs en acides aminés des régimes sont estimées à partir des valeurs obtenues sur les matières premières, par chromatographie sur échangeurs d'ions. Les acicles aninés libres du sang sont extraits par épuisements successifs par l'éthanol 82 p. 10o, puis dosés par chromatographic sur colonne, sur appareil automatique, suivant une méthodologie décrite par ailleurs (DUEE et RÉRAT, I 975 ).

\section{RÉSULTATS}

$$
\text { 1. - Croissance et consommation (tabl. I) }
$$

Une supplémentation du régime de base par la lysine seule (lot 2) ne procure qu'une légère amélioration, d'ailleurs non significative, de la vitesse de croissance $(33+\mathrm{g} / \mathrm{j}$ contre 308$)$ et de l'indice de consommation $(3,40$ contre 3,99). [n apport supplémentaire de thréonine (Iot 3) est

(1) Composition p. roo: tourteau d'arachide, I6; gluten de maïs "globazote ", I 2 ; anidon de mais, $5 \mathrm{I}$; huile de mais, 5 ; cellulose de bois tibreuse purifice $\mathrm{I} 2$; mélange minéral, 3 dont $\mathrm{I}, 6$ de phosphate bicalcique, o,95 de craie broyée, o,35 de sel marin et o, 10 d'un mélange d'oligoćléments (Hen r r ot al., I 975 ); mélange vitaminique (HENR' et al., r 975 ), $\mathrm{I}, \mathrm{O}$ 
sans effet sur la vitesse de croissance $(320 \mathrm{~g} / \mathrm{j})$ comme sur l'indica de consommation (4,oz). Par contre, une double supplémentation en lysine et en tryptophane (lot 4) entraîne une amólioration significative du gain moyen journalier $(+\mathrm{I} 89 \mathrm{~g})$, accompagné a d'une élévation du niveau d'ingestion de nourriture ( $\mathrm{I}, 44 \mathrm{~kg} / \mathrm{j}$ contre $\mathrm{I}, \mathrm{I} 6$ dans le lot $\mathrm{I}$ ) et d'une nette diminution de l'indice de consommation (- 1,05$)$.

\section{TABLEAU I}

Résultats de croissance et de consommation ( $\left.{ }^{1}\right)$

Growth performance

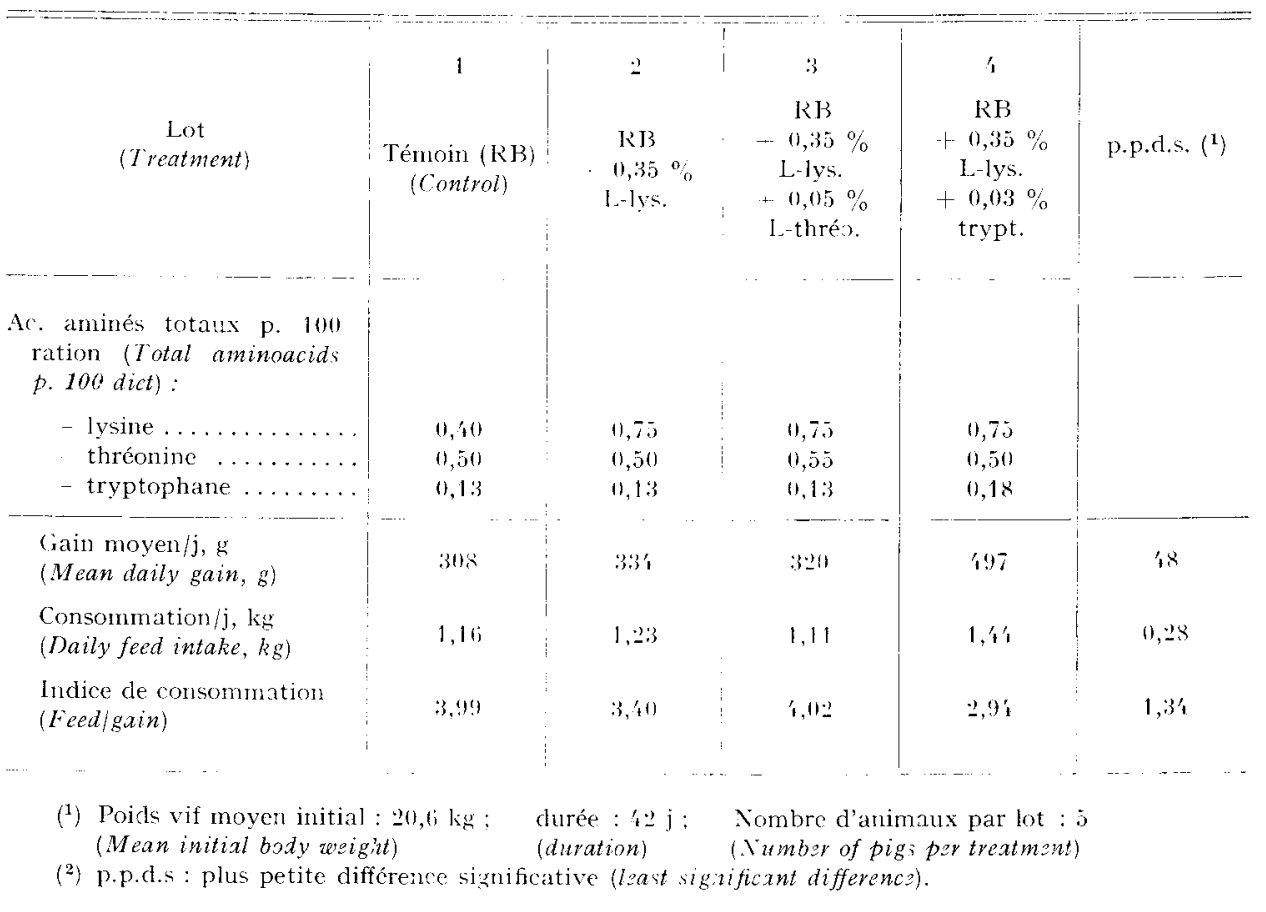

2. ..... Teneurs en acides aminés libres du sang (tabl. 2)

Le tryptophane a été dosé en utilisant la même colonne d'extraction que pour les autres acides aminés. En raison vraisemblablement des pertes de tryptophane entraînés par cette méthode, les résultats doivent ainsi être considérés avec une certaine prudence, tout au moins en valeur absolue. L'addition d'une surcharge de tryptophanc à un échantillon də sang $(5,03 \mathrm{~g} / \mathrm{r} 00 \mathrm{~g})$ a permis cependant une récupération de 65 p. 100.

L'addition de lysine seule au régime de base (lot 2) provoque une nette diminution de la thréonine et du tryptophane libres dans le sang, correspondant à une meilleure utilisation de ces acides aminés. Il en est de même de la plupart des autres acides aminés indisponsảbles, notamment la valine, la méthionine, l’isoleucine, la phénylalanine et la tyrosine, ce qui sa traduit par une valeur plus faible de la somme des acides aminés indispensables libres du sang ( $2 \mathrm{AAI}$ ), soit I9,5 g pour Ioo g de sang contre 22, I dans le lot $\mathrm{I}$.

Par rapport au lot 2 supplémenté en lysine, un apport supplémentaire de thréonine provoque une élévation de la somme des acides aminés libres dosés (indispensables et non indispensables) 
parmi lesquels la thréonine et la lysine qui se trouvent ainsi en excés par rapport aux besoins.

Dans le lot 4 , un apport supplémentaire de tryptophane se traduit par rapport au lot 2 simplement supplémenté en lysine, par une augmentation de la teneur en cet acide aminé dans le sang. En ce qui concerne les autres acides aminés indispensables, a l'exception de la lysine et de l'arginine, leurs concentrations dans le sang sont abaissées. Il en est ainsi de la méthionine et de la cystine, de l'isoleucine, de la leucine, de la phénylalanine et de la tyrosine, ainsi que de I'histidine, ce qui semble indiquer une meilleure utilisation dé ces acides aminés à la suite d'une supplémentation en tryptophanc.

\section{TABIEAT 2}

Acides amines libris du sang (on meloog g somg frais) Blosd fiet anino acids (mpl noo

Ac. aninés totaux p. 100 ration

(Total aminoacids p. 100 diet)

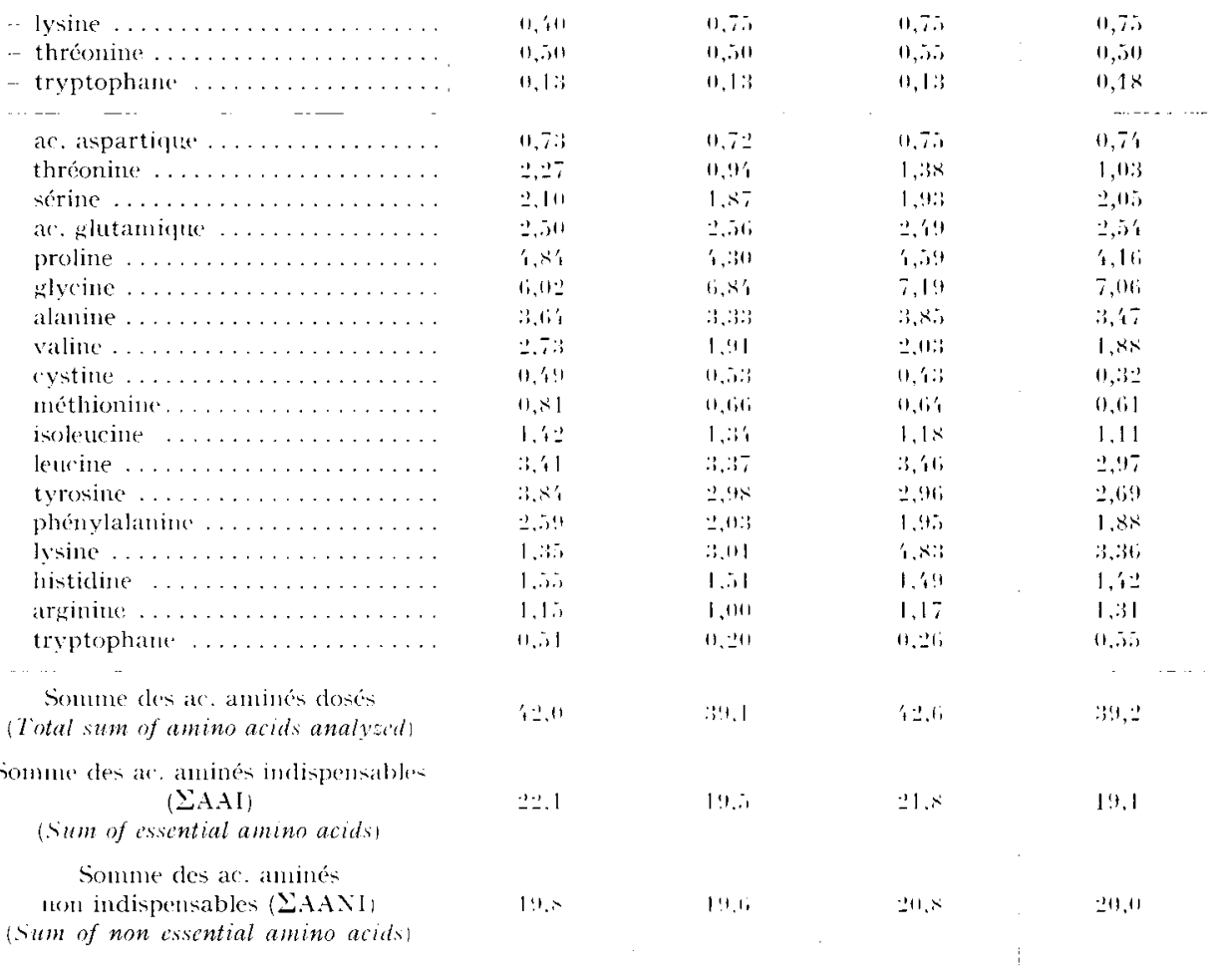


D'une manière générale, il est à remarquer que les tencurs en acides aminés libres dans le sang sont plus faibles que celles enregistrées antérieurement avec des animaux au même stade physiologique (Prox et al., i971; Hexry et al., 1975). Cette différence s'explique par un temps cle prélèvement du sang plus long après le repas, soit 8 heures au lieu de 4 et $5 \mathrm{~h} 30$.

\title{
CONCLUSION
}

Les résultats de la présente étude ont fait apparaître un déficit secondaire en tryptophane clans un régime renfermant i 6 p. Ioo de protéines fournies en parties égales par le tourteau d'arachicle et le gluten de maïs. La correction de ce déficit entraîne une amélioration du gain moyen journalier de près de $50 \mathrm{p}$. Ioo, tandis que le niveau d'ingestion est accru de $17 \mathrm{p}$. I0o. Comme le Rat et le Poulet, le Pore manifeste ainsi une grande sensibilité à un déséquilibre en acides aminés dans sa ration.

La modification du profil cles acides aminés libres sanguins constitue une donnée caractéristique associée à l'effet dépressif d'un déséquilibre en acides aminés sur l'appétit, probablement par l'intermédiaire des centres régulateurs hypothalamiques, ce qui confirme les nombreuses observations faites sur ce sujet chez les autres espèces animales, notamment le Rat et le Poulet.

Recu pour publication en juillet 1975.

\section{SUMMARY}

\author{
EIFECT OF TRYPTOPHAN DEFICIENCY IN THE PIG DIE'T \\ ON FEEI INTAKE AND GROWTH PERFORMANCE
}

A trial of six week duration was conclucted on growing pigs, weighing initially $20,6 \mathrm{~kg}$ and fed a semi-purified diet composed of peanut meal and gluten meal. After supplementing with a normal dose of dietary lysine, a tryptophan deficiency was observed, with depressive effects on fecd intake and growth performance, as well as a modification in the blood free amino acidemia.

\section{RÉFÉRENCES BIBLIOGRAPHIQUES}

DUee P. H., Rérat A., 1975 . Étude du besoin en lysine de la truie gestante nullipare. Ann. Zootech,

24, 447-464.

Harper A. E., I964. Amino acid toxicities and imbalances. In Munro H. N. et Allison J. B., Mammatian protein metabolism., Vol. II, 87, Acad. Press. New York.

HENRY Y., DUEe P. H., RÉRAT A., 1976. Isoleucine requirement of the growing pig and leucineisoleucine interrelationship. J. anim. sci., (in press).

Mahan D. C., Wilson R. F., Sharda D. P., I973. Limiting anino acids in low protein corn-soybean meal diets for pigs. J. anim. sci., 37, 286 (abstr.).

Múller Z., Kozer V., Bauer B., Strunc M., Moravec J., 1967 . Enrichissement des diètes céréalières, destinées à l'engraissement des porcs, depuis $35 \mathrm{~kg}$ de poids vif, en lysine, thréonine, tryptophane et méthionine, (Tchèque). Biologizace a chemizace, 5, 399-410.

N. R. C., r973. Nutrient requirements of swine, 56 p. Nat. Acad. Sci., N. R. C., Washington D. C. 
Ptox R., Prugnadd J., Henry Y., Rérat A., ig7r. Infuence de ha teneur en thréonine du régime sur l'aminoacidémie libre du porc en croissance. X' Congrès Internat. Zootech., Versailles.

Sugahara M., Bakir D. H., Harmox B. (i, Jensex A. H., r969. Eiffect of excess levels of dietary protein on carcass development in swine. J. anim. sci., 29, 598-60r.

Ziмmermax R. A., ScotT H. M., ig65. Interrelationship of plasma amino acid levels and weight gain in the chick as influenced by suboptinal and superoptinal dietary concentrations of single anino acids. J. Nutr., 87, I 3-18. 